\title{
ON A LIMIT PASS FROM TWO-POINT TO ONE-POINT INTERACTION IN A ONE DIMENSIONAL QUANTUM MECHANICAL PROBLEM GIVING RISE TO A SPONTANEOUS SYMMETRY BREAKING
}

\author{
A. Restuccia ${ }^{1,2}$, A. Sotomayor ${ }^{1}$, V.A. Strauss St3 $^{2,3}$ \\ ${ }^{1}$ Universidad de Antofagasta, Antofagasta, Republic of Chile \\ ${ }^{2}$ Universidad Simón Bolívar, Caracas, Venezuela \\ ${ }^{3}$ Ulyanovsk State Pedagogical University, Ulyanovsk, Russian Federation \\ E-mails: alvaro.restuccia@uantof.cl, adrian.sotomayor@uantof.cl,vstrauss@mail.ru
}

\begin{abstract}
We analyze, by means of singular potentials defined in terms of Dirac functions and their derivatives, a one dimensional symmetry breaking in quantum mechanics. From a mathematical point of view we use a technique of selfadjoint extensions applied to a symmetric differential operator with a domain containing smooth functions which vanish at two inner points of the real line. As is well known, the latter leads to a two-point boundary problem. We compute the resolvent of the corresponding extension and investigate its behavior in the case in which the inner points change their positions. The domain of these extensions can contain some functions with non differentiability or discontinuity at the points mentioned before. This fact can be interpreted as a presence of singular potentials like shifted Dirac delta functions and/or their first derivative centered at the same points. Then, we study the existence of broken-symmetry bound states. For some given entanglement boundary conditions we can show the existence of a ground state, which leads to a spontaneous symmetry breaking. We also prove that within a frame of Pontryagin spaces this type of symmetry breaking is saved if the distance between the mentioned above interior points tends to zero and then we can reformulate this result in terms of a larger Hilbert space.
\end{abstract}

Keywords: operator theory; resolvent; solution of wave equation: bound states; spontaneous symmetry breaking; Pontryagin spaces.

\section{Introduction}

This work is a direct continuation of the papers [1] and [2]. In particular, [2] contains our detailed motivation to the present studies and a sketch concerning the history of corresponding problems (see also [3] and [4], for the present state of this approach see [5] and [6] ). Because of this, we not mention here the related historical aspects. We consider the spontaneous symmetry breaking (see [7] and [8]) in one dimensional quantum mechanical problems in terms of two-point boundary problems with entanglement which leads to singular potentials (the derivative of two tied shifted delta-functions).

In Section 1 we recall some well known results concerning a Hamiltonian with onepoint interaction. In Section 2 we present some results on the study of singular potentials in terms of shifted delta-functions or their first derivative. In particular, we discuss a Hamiltonian (containing a two-point interaction) whose ground state is degenerate, with two eigenfunctions. Next, in Section 3 we go to the main contribution of the paper using different forms of limit pass from two-point interaction to one-point one. In particular, a selfadjoint extension of a limit differential operator to a larger Pontryagin space preserves an option of degenerate ground state, moreover, the corresponding Hamiltonian contains the second derivative of delta-function. Finally, we give our conclusions in Section 4. 


\section{Prologue}

Our principal aim is a pass from two-point interaction to one-point one, so we need to recall some basic facts concerning one-point interaction. Let the differential operator $D_{0}=-d^{2} \cdot / d x^{2}$ have the domain

$$
\mathcal{D}\left(D_{0}\right)=\left\{y(x) \mid y(x) \in W^{2,2}(\mathbb{R}), y(0)=y^{\prime}(0)=0\right\},
$$

where $W^{2,2}(\mathbb{R})$ is the corresponding Sobolev space. Its adjoint one $D_{0}^{*}$ has the domain

$$
\mathcal{D}\left(D_{0}^{*}\right)=\left\{y(x)|y(x)|_{\mathbb{R}_{+}} \in W^{2,2}\left(\mathbb{R}_{+}\right),\left.y(x)\right|_{\mathbb{R}_{-}} \in W^{2,2}\left(\mathbb{R}_{-}\right)\right\},
$$

where $\mathbb{R}_{+}=\{x \mid x>0\}$ and $\mathbb{R}_{-}=\{x \mid x<0\}$, so $x(t), x^{\prime}(t)$ are absolutely continuous functions in the both open half-lines $\mathbb{R}_{-}$and $\mathbb{R}_{+}$, but, generally speaking, are not defined at zero. At the same time, the following left- and right-hand limits $y(-0)=\lim _{x \rightarrow 0, x<0} y(x)$, $y^{\prime}(-0)=\lim _{x \rightarrow 0, x<0} y^{\prime}(x), y(+0)=\lim _{x \rightarrow 0, x>0} y(x)$ and $y^{\prime}(+0)=\lim _{x \rightarrow 0, x>0} y^{\prime}(x)$ are well defined.

Evidently $D_{0} \subset D_{0}^{*}$, so $D_{0} \subset \widetilde{D}_{0} \subset D_{0}^{*}$ for every selfadjoint extension $\widetilde{D}_{0}$ of $D_{0}$. Thus, any extension of $D_{0}$ can be obtained as a restriction of $D_{0}^{*}$. A simple calculation yields $\left(D_{0}^{*} y, z\right)=-y^{\prime}(-0) \bar{z}(-0)+y^{\prime}(+0) \bar{z}(+0)+y(-0) \bar{z}^{\prime}(-0)-y(+0) \bar{z}^{\prime}(+0)+\left(y, D_{0}^{*} z\right)$. Therefore, any selfadjoint restriction of $D_{0}^{*}$ must be such that

$$
y^{\prime}(+0) \bar{z}(+0)-y^{\prime}(-0) \bar{z}(-0)-y(+0) \bar{z}^{\prime}(+0)+y(-0) \bar{z}^{\prime}(-0)=0 .
$$

Any set of boundary values $\left(y(-0), y^{\prime}(-0), y(+0), y^{\prime}(+0)\right)$ can be considered as an element of a four-dimensional pseudo-unitary space (for the terminology see [9]) and Equality (3) means that for any selfadjoint restriction the corresponding sets must form a twodimensional neutral subspace. Alternatively, for a selfadjoint restriction one need to define two suitable linear homogenous conditions. Some of these accept jumps at zero for $y(x)$ and/or $y^{\prime}(x)$. The latter is well known (see the book [5]) and can be interpreted as the Hamiltonian with one-point interaction involving delta-function and/or its first derivative. In [5] also singular potentials with derivatives of the shifted delta-function in finitely many points were discussed, but this discussion was restricted to local boundary conditions only. For historical details see also [6]. Within the frame of one-point problem there are some selfadjoint extensions with two negative eigenvalues [10]: for $\widetilde{D}_{0}$ given by the boundary conditions $(\alpha>0, \beta>0)$

$$
\begin{aligned}
& \alpha(y(+0)+y(-0))=\left(-y^{\prime}(+0)+y^{\prime}(-0)\right), \\
& \beta(y(+0)-y(-0))=-\left(y^{\prime}(+0)+y^{\prime}(-0)\right),
\end{aligned}
$$

conditions (3) are fulfilled, so it is selfadjoint. This extension has the eigenvalues $-\alpha^{2}$ and $-\beta^{2}$ with the eigenfunctions $e^{-\alpha|x|}$ and $\operatorname{Sgn}(x) e^{-\beta|x|}$ respectively $(\operatorname{Sgn}(x)=-1$ for $x<0$, $\operatorname{Sgn}(0)=0, \operatorname{Sgn}(x)=1$ for $x>0)$ and $\widetilde{D}_{0} y(x)=-y^{\prime \prime}(x)-\frac{1}{\beta} \cdot \delta^{\prime}(x)\left(y^{\prime}(-0)+y^{\prime}(+0)\right)-$ $\alpha \cdot \delta(x)(y(-0)+y(+0))$.

Let $\alpha=\beta$. The latter means that $\widetilde{D}_{0}$ has the unique negative eigenvalue $-\alpha^{2}$ with two non symmetric eigenfunctions

$$
y_{1}(t)=\left\{\begin{array}{l}
e^{\alpha x}, \text { if } x<0, \\
0, \text { if } x>0
\end{array} \quad \text { and } y_{2}(x)=\left\{\begin{array}{l}
0, \text { if } x<0, \\
e^{-\alpha x}, \text { if } x>0 .
\end{array}\right.\right.
$$


The case in question does not represent a model related with a spontaneous symmetry breaking. Indeed, the latter is the case of a non transitable barrier: Conditions (4) imply $\alpha \cdot y(-0)=y^{\prime}(-0)$ and $\alpha \cdot y(+0)=-y^{\prime}(+0)$, so the waves with support on $\mathbb{R}_{-}$and $\mathbb{R}_{+}$ are independent. Selfadjoint extensions of this type were named in [6] separated.

In next sections we will consider some analogous schemes for boundary problems at two interior points $-h, h$ and a behavior of the corresponding extensions if $h \rightarrow 0$.

\section{Two-Point Interaction}

\subsection{An Underlying Idea}

Let the differential operator $D_{h}=-d^{2} \cdot / d x^{2}$ have the domain $\mathcal{D}\left(D_{h}\right)=\{y(x) \mid y(x) \in$ $\left.W^{2,2}(\mathbb{R}), y( \pm h)=y^{\prime}( \pm h)=0\right\}$. Then the adjoint operator $D_{h}^{*}$ has the domain

$$
\mathcal{D}\left(D_{h}^{*}\right)=\left\{y(x)|y(x)|_{\mathbb{R}_{-h}} \in W^{2,2}\left(\mathbb{R}_{-h}\right),\left.y(x)\right|_{(-h, h)} \in W^{2,2}((-h, h)),\left.y(x)\right|_{\mathbb{R}_{h}} \in W^{2,2}\left(\mathbb{R}_{h}\right)\right\},
$$

where $\mathbb{R}_{-h}=(-\infty,-h), \mathbb{R}_{h}=(h,+\infty)$. A restriction of $D_{h}^{*}$ is selfadjoint for any collection of four linearly independent homogenous boundary conditions that yields

$$
\begin{aligned}
& -y^{\prime}(-h-0) \bar{z}(-h-0)+y^{\prime}(-h+0) \bar{z}(-h+0)-y^{\prime}(h-0) \bar{z}(h-0)+ \\
& +y^{\prime}(h+0) \bar{z}(h+0)+y(-h-0) \bar{z}^{\prime}(-h-0)-y(-h+0) \bar{z}^{\prime}(-h+0)+ \\
& +y(h-0) \bar{z}^{\prime}(h-0)-y(h+0) \bar{z}^{\prime}(h+0)=0
\end{aligned}
$$

Using this way one can, for example, put

$$
y(-h-0)=y(-h+0), \quad y(h-0)=y(h+0),
$$

and the same for $z(x)$, that yields the continuity of $y$ and $z$. The latter converts (5) to

$$
\begin{aligned}
& \left(y^{\prime}(-h+0)-y^{\prime}(-h-0)\right) \bar{z}(-h)+\left(y^{\prime}(h+0)-y^{\prime}(h-0)\right) \bar{z}(h)- \\
& -y(-h)\left(\bar{z}^{\prime}(-h+0)-\bar{z}^{\prime}(-h-0)\right)-y(h)\left(\bar{z}^{\prime}(h+0)-\bar{z}^{\prime}(h-0)\right)=0 .
\end{aligned}
$$

An important part of this case (including an entanglement of boundary conditions) was analyzed in [1]. In particular, it was shown that under some restrictions and $h \rightarrow 0$ Conditions (6), (7) convert to Conditions (4).

Next, let $D_{\text {reg }}$ be the selfadjoint differential operator given by the formal differential expression $D_{\text {reg }}=-d^{2} \cdot / d t$ with the domain $\mathcal{D}\left(D_{\text {reg }}\right)=\left\{y(t) \mid y(t) \in W^{2,2}(\mathbb{R})\right\}$. Let

$$
G(t)=\frac{e^{-\gamma|t|}}{2 \gamma}
$$

Then for every $z(t) \in L^{2}(\mathbb{R})$ and $\gamma>0$

$$
\left(\gamma^{2} I+D_{\text {reg }}\right)^{-1} z(t)=\int_{-\infty}^{+\infty} z(\tau) G(t-\tau) d \tau
$$

Note that $D_{\text {reg }}$ is the restriction of $D_{h}^{*}$ generated (see (5)) by the boundary conditions

$$
\begin{aligned}
& y(-h-0)=y(-h+0), \quad y(h-0)=y(h+0), \\
& y^{\prime}(-h-0)=y^{\prime}(-h+0), \quad y^{\prime}(h-0)=y^{\prime}(h+0) .
\end{aligned}
$$




\subsection{An Entanglement of Boundary Conditions: Continuous First Derivative}

In this subsection we assume $y^{\prime}(-h-0)=y^{\prime}(-h+0), y^{\prime}(h-0)=y^{\prime}(h+0)$ and the same for $z(x)$. Then the conditions of selfadjointness for restrictions of $D_{h}^{*}$ takes the form

$$
\begin{aligned}
& y^{\prime}(-h)(-\bar{z}(-h-0)+\bar{z}(-h+0))+y^{\prime}(h)(-\bar{z}(h-0)+\bar{z}(h+0))+ \\
& +(y(-h-0)-y(-h+0)) \bar{z}^{\prime}(-h)+(y(h-0)-y(h+0)) \bar{z}^{\prime}(h)=0,
\end{aligned}
$$

where $y^{\prime}(-h)=y^{\prime}(-h \pm 0), y^{\prime}(h)=y^{\prime}(h \pm 0)$. Let additionally

$$
\left(\begin{array}{c}
(y(-h+0)-y(-h-0)) \\
(y(h+0)-y(h-0))
\end{array}\right)=\left(\begin{array}{ll}
b_{11} & b_{12} \\
b_{21} & b_{22}
\end{array}\right)\left(\begin{array}{c}
y^{\prime}(-h) \\
y^{\prime}(h)
\end{array}\right) .
$$

The symmetry of the matrix $B=\left(\begin{array}{ll}b_{11} & b_{12} \\ b_{21} & b_{22}\end{array}\right)$ is equivalent to the selfadjointness of the corresponding restriction. If one considers $y(x)$ under conditions (11) as a generalized function (distribution), then $y^{\prime \prime}(x)=y_{c l}^{\prime \prime}(x)+\left(b_{11} y^{\prime}(-h)+b_{12} y^{\prime}(h)\right) \delta^{\prime}(x+h)+\left(b_{21} y^{\prime}(-h)+\right.$ $\left.b_{22} y^{\prime}(h)\right) \delta^{\prime}(x-h)$, where $f_{c l}^{\prime \prime}(x)=f^{\prime \prime}(x)$ if $f^{\prime \prime}(x)$ exists in the classical sense and $f_{c l}^{\prime \prime}(x)=0$ in the opposite case. Then the corresponding extension $\widetilde{D}_{h}$ of $D_{h}$ can be re-written as

$$
\begin{aligned}
\widetilde{D}_{h} y(x)=-y_{c l}^{\prime \prime}(x)= & -y^{\prime \prime}(x)+\left(b_{11} y^{\prime}(-h)+b_{12} y^{\prime}(h)\right) \delta^{\prime}(x+h)+ \\
& +\left(b_{21} y^{\prime}(-h)+b_{22} y^{\prime}(h)\right) \delta^{\prime}(x-h) .
\end{aligned}
$$

Let us choose a matrix $B$ such that for every positive $h$ the functions

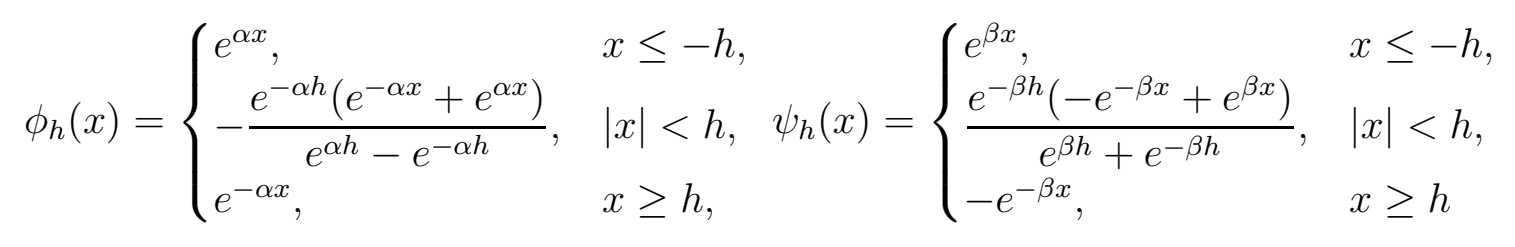

would be eigenfunctions of the operator $\widetilde{D}_{h}$. Then

$$
\begin{aligned}
& b_{22}=b_{11}=-\left(\frac{1}{\alpha\left(1-e^{-2 \alpha h}\right)}+\frac{1}{\beta\left(1+e^{-2 \beta h}\right)}\right), \\
& b_{21}=b_{12}=\left(\frac{1}{\alpha\left(1-e^{-2 \alpha h}\right)}-\frac{1}{\beta\left(1+e^{-2 \beta h}\right)}\right) .
\end{aligned}
$$

Note that $\int_{-h}^{h} \phi_{h}(x) d x=-\frac{2 e^{-\alpha h}}{\alpha}$, so in the sense of distributions

$$
\lim _{h \rightarrow+0} \phi_{h}(x)=\phi_{0}(x)-\frac{2}{\alpha} \delta(x)
$$

where $\phi_{0}(x)=e^{-\alpha|x|}$, therefore in this case the limit generates a new boundary problem, that (maybe!) directly involves $\delta(x)$.

The extension of $D_{h}$ corresponding to (13) will be denote by $\widehat{D}_{h}$. Then (see (12))

$$
\begin{aligned}
\widehat{D}_{h} y(x)=-y^{\prime \prime}(x)+ & \frac{\left(\delta^{\prime}(x-h)-\delta^{\prime}(x+h)\right)\left(y^{\prime}(-h)-y^{\prime}(h)\right)}{\alpha\left(1-e^{-2 \alpha h}\right)}- \\
& -\frac{\left(\delta^{\prime}(x+h)+\delta^{\prime}(x-h)\right)\left(y^{\prime}(-h)+y^{\prime}(h)\right)}{\beta\left(1+e^{-2 \beta h}\right)} .
\end{aligned}
$$


It is unclear how to treat a limit pass by $h \rightarrow 0$ in the latter expression: the domain of $\widehat{D}_{h}$ depends on $h$ and the eigenfunction $\phi_{h}(x)$ does not converge to any function in $L^{2}(\mathbb{R})$. In the next section we consider some different approaches to this problem.

\section{A Limit Pass to One-Point Interaction:}

\subsection{A Traditional Approach}

In this Subsection we describe a suitable expression for the resolvent of $\widehat{D}_{h}$ for a negative number $-\gamma^{2}, \gamma>\alpha, \gamma>\beta$ and estimate the resolvent behavior for $h \rightarrow 0$.

Both operators $\widehat{D}_{h}$ and $D_{\text {reg }}$ are restrictions of $D^{*}$. Moreover (compare Conditions (10) and $(11)), y(t) \in \widehat{D}_{h} \cap D_{\text {reg }}$ if and only if $y(t) \in W^{2,2}(\mathbb{R})$ and $y^{\prime}(-h)=y^{\prime}(h)=0$. Using this reasoning and (9) one can proves (see [2] for details) that $\left(\gamma^{2} I+\widehat{D}_{h}\right)^{-1} f(t)=$

$$
\begin{aligned}
& \left(\int_{-\infty}^{+\infty} f(\tau) G(t-\tau) d \tau-\left(\frac{\int_{-\infty}^{+\infty} \phi_{h}(\tau) G(t-\tau) d \tau}{\eta_{h}} \int_{-\infty}^{+\infty} f(t) s_{h}(t) d t+\right.\right. \\
& \left.\left.+\frac{\int_{-\infty}^{+\infty} \psi_{h}(\tau) G(t-\tau) d \tau}{\theta_{h}} \int_{-\infty}^{+\infty} f(t) w_{h}(t) d t\right)\right)+ \\
& +\left(\frac{\phi_{h}(t)}{\left(\gamma^{2}-\alpha^{2}\right) \eta_{h}} \int_{-\infty}^{+\infty} f(t) s_{h}(t) d t+\frac{\psi_{h}(t)}{\left(\gamma^{2}-\beta^{2}\right) \theta_{h}} \int_{-\infty}^{+\infty} f(t) w_{h}(t) d t\right),
\end{aligned}
$$

where

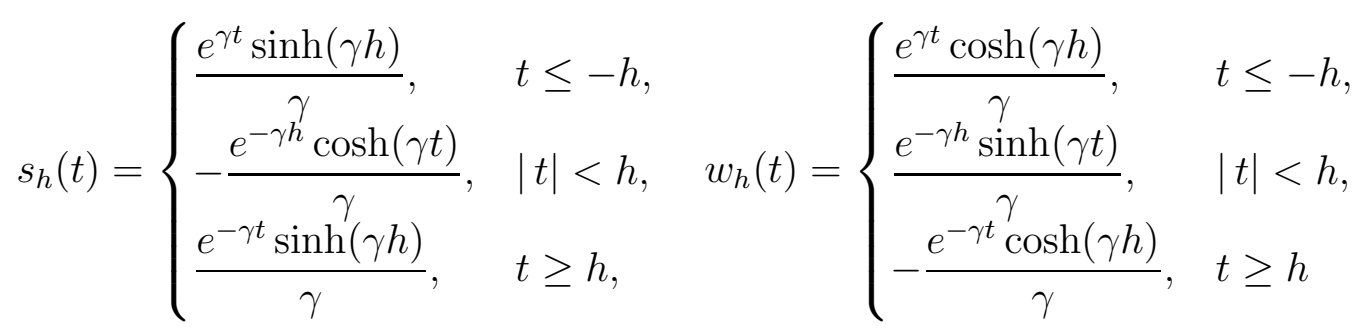

and

$$
\begin{aligned}
& \eta_{h}=2 \frac{\sinh (\gamma h)}{\gamma} \cdot \frac{e^{-(\alpha+\gamma) h}}{\alpha+\gamma}+\frac{e^{-(\alpha+\gamma) h}}{\gamma \sinh (\alpha h)} \cdot\left\{\frac{\sinh (\alpha+\gamma) h}{(\alpha+\gamma)}+\frac{\sinh (\gamma-\alpha) h}{(\gamma-\alpha)}\right\}, \\
& \theta_{h}=2 \frac{\cosh (\gamma h)}{\gamma} \cdot \frac{e^{-(\beta+\gamma) h}}{\beta+\gamma}+\frac{e^{-(\beta+\gamma) h}}{\gamma \cosh (\beta h)} \cdot\left\{\frac{\sinh (\beta+\gamma) h}{(\beta+\gamma)}-\frac{\sinh (\gamma-\beta) h}{(\gamma-\beta)}\right\} .
\end{aligned}
$$

Remark 1. Formulae (16), (17), (18) (19) show that al spectrum of $\widehat{D}_{h}$, except for two points $-\alpha^{2}$ and $-\beta^{2}$, is non negative.

In [2] the following equalities were proved

$$
\lim _{h \rightarrow 0}\left\|\phi_{h}(t) \cdot \int_{-\infty}^{+\infty} f(t) s_{h}(t) d t\right\|=0,
$$




$$
\begin{aligned}
& \lim _{h \rightarrow 0}\left(\gamma^{2} I+\widehat{D}_{h}\right)^{-1} f(t)=\int_{-\infty}^{+\infty} f(\tau) G(t-\tau) d \tau+ \\
& \left(\frac{2 \gamma^{2} G(t) \operatorname{Sgn}(t)}{(\gamma-\beta)} \int_{-\infty}^{+\infty} f(t) G(t) \operatorname{Sgn}(t) d t\right) .
\end{aligned}
$$

Thus, the strong limit $s-\lim _{h \rightarrow 0}\left(\gamma^{2} I+\widehat{D}_{h}\right)^{-1}$ is a one-dimensional perturbation of the resolvent for $D_{\text {reg }}$ (see (9)). If $f(t)$ is even, then (9) and (21) bring $\lim _{h \rightarrow 0}\left(\gamma^{2} I+\widehat{D}_{h}\right)^{-1} f(t)=\left(\gamma^{2} I+\right.$ $\left.D_{\text {reg }}\right)^{-1} f(t)$ but $D_{\text {reg }}$ has not a point spectrum. Thus, the operator-limit lost the eigenvalue $\frac{1}{\gamma^{2}-\alpha^{2}}$ and the corresponding even eigen-function.

Remark 2. The function $\psi_{0}(t)=e^{-\beta|t|} \operatorname{Sgn}(t)$ is an eigenfunction for $\lim _{h \rightarrow 0}\left(\gamma^{2} I+\widehat{D}_{h}\right)^{-1}$ corresponding to the eigenvalue $\frac{1}{\left(\gamma^{2}-\beta^{2}\right)}$. The unbounded operator $\left(s-\lim _{h \rightarrow 0}\left(\gamma^{2} I+\widehat{D}_{h}\right)^{-1}\right)^{-1}-\gamma^{2} I$ is a selfadjoint restriction of $D_{0}^{*}$ (see (2) and (3)) corresponding to the boundary conditions

$$
y^{\prime}(-0)=y^{\prime}(+0),-\beta(y(+0)-y(-0))=y^{\prime}(-0)+y^{\prime}(+0),
$$

that is a particular case of (4) with $\alpha=0$.

Proof. The statement concerning $\psi_{0}(t)$ can be checked directly, so it is enough to show that (21) brings $(22)$ and viceversa. Let $\omega(t)=\frac{1}{\gamma^{2}-\beta^{2}}\left(2 \gamma G(t)-e^{-\beta|t|}\right) \operatorname{Sgn}(t), f(t) \in L^{2}(\mathbb{R})$, $x(t)=\int_{-\infty}^{+\infty} f(\tau) G(t-\tau) d \tau+\gamma(\beta+\gamma) \omega(t) \int_{-\infty}^{+\infty} f(t) G(t) \operatorname{Sgn}(t) d t$. Then $\omega(t)$ is continuous and has an absolutely continuous derivative: $\omega^{\prime}(t)=-\left(\frac{\gamma e^{-\gamma|t|}-\beta e^{-\beta|t|}}{\gamma^{2}-\beta^{2}}\right)$, so $x^{\prime}(0)=0$. Thus, $x(t)$ satisfies $(22)$. Now let $y(t) \in \mathcal{D}\left(D_{0}^{*}\right)$ and satisfy (22). One need to prove that there is $f(t) \in L^{2}(\mathbb{R})$ such that $\lim _{h \rightarrow 0}\left(\gamma^{2} I+\widehat{D}_{h}\right)^{-1} f(t)=y(t)$ and $\left(D_{0}^{*}+\gamma^{2} I\right) y(t)=f(t)$. Let $\tilde{y}(t)=y(t)+\frac{y(+0)-y(-0)}{2} \psi_{0}(t)$ for $t \neq 0$ and $\tilde{y}(0)=\frac{y(-0)+y(+0)}{2}$. Then $\tilde{y}(t)$ is absolutely continuous function, its derivative is absolutely continuous too and due to $(22) y^{\prime}(0)=0$. Put $f(t)=\left(\tilde{y}^{\prime \prime}(t)+\gamma^{2} \tilde{y}(t)\right)-\frac{(y(+0)-y(-0))\left(\gamma^{2}-\beta^{2}\right)}{2} \psi_{0}(t)$. Note that $\tilde{y}(t) \in \mathcal{D}\left(D_{\text {reg }}\right)$, so $\int_{-\infty}^{+\infty}\left(\tilde{y}^{\prime \prime}(\tau)+\gamma^{2} \tilde{y}(\tau)\right) G(t-\tau) d \tau=\tilde{y}(t)$. The rest is trivial.

\subsection{An Extension on a Larger Space}

Let us consider the term $\phi_{h}(t) \cdot \int_{-\infty}^{+\infty} f(t) s_{h}(t) d t$ for an arbitrary function $f(t) \in$ $L^{2}(\mathbb{R})$ continuous at zero and find its limit in point-wise sense. If $t_{0} \neq 0$, then 


$$
\begin{aligned}
\lim _{h \rightarrow 0} \phi_{h}\left(t_{0}\right) \cdot \int_{-\infty}^{+\infty} f(t) s_{h}(t) d t & =\lim _{h \rightarrow 0} e^{-\alpha\left|t_{0}\right|} \cdot \int_{-\infty}^{+\infty} f(t) s_{h}(t) d t=0, \text { but } \\
\lim _{h \rightarrow 0} \phi_{h}(0) \cdot \int_{-\infty}^{+\infty} f(t) s_{h}(t) d t & =-\frac{1}{\alpha} \int_{-\infty}^{+\infty} f(t) e^{-\gamma|t|} d t+\frac{2}{\gamma \alpha} f(0) .
\end{aligned}
$$

The function

$$
\iota(t)= \begin{cases}0, & \text { if } t \neq 0 \\ 1, & \text { if } t=0\end{cases}
$$

has no sense as an element of $L^{2}(\mathbb{R})$, but can be a "legal" element in a larger space. Let

$$
\sigma_{0}(t)= \begin{cases}t-\frac{1}{2}, & \text { if } t \leq 0 \\ t+\frac{1}{2}, & \text { if } t>0\end{cases}
$$

Then $\iota(t) \in L_{\sigma_{0}}^{2}(\mathbb{R})$ and $\|\iota(t)\|=1$. Let us introduce the orthoprojection $\left(P_{0} g\right)(t)$ :

$$
\left(P_{0} g\right)(t)=g(0) \iota(t), \quad g(t) \in L_{\sigma_{0}}^{2}(\mathbb{R}) .
$$

Note that for any $g(t) \in L_{\sigma_{0}}^{2}(\mathbb{R})$ :

$$
\left\|\left(I-P_{0}\right) g(t)\right\|_{L_{\sigma_{0}}^{2}(\mathbb{R})}=\|g(t)\|_{L^{2}(\mathbb{R})} .
$$

Let us introduce the operator $A_{h}$ (compare with $\left.(16)\right): L^{2}(\mathbb{R}) \cap \mathbf{C}(\mathbb{R}) \Rightarrow L_{\sigma_{0}}^{2}(\mathbb{R})$,

$$
\begin{aligned}
& \left(A_{h} f\right)(t)=\left(\int_{-\infty}^{+\infty} f(\tau) G(t-\tau) d \tau-\left(\frac{\int_{-\infty}^{+\infty} \phi_{h}(\tau) G(t-\tau) d \tau}{\eta_{h}} \int_{-\infty}^{+\infty} f(t) s_{h}(t) d t+\right.\right. \\
& \left.\left.+\frac{\int_{-\infty}^{+\infty} \psi_{h}(\tau) G(t-\tau) d \tau}{\theta_{h}} \int_{-\infty}^{+\infty} f(t) w_{h}(t) d t\right)\right)+ \\
& +\left(\frac{\phi_{h}(t)}{\left(\gamma^{2}-\alpha^{2}\right) \eta_{h}} \int_{-\infty}^{+\infty} f(t) s_{h}(t) d t+\frac{\psi_{h}(t)}{\left(\gamma^{2}-\beta^{2}\right) \theta_{h}} \int_{-\infty}^{+\infty} f(t) w_{h}(t) d t\right) .
\end{aligned}
$$

Note that for any $f(t) \in L^{2}(\mathbb{R}) \cap \mathbf{C}(\mathbb{R})$ the corresponding function $\left(A_{h} f\right)(t)$ is continuous at zero, so (27), evenness of $G(t)$ (see (8)) and oddness of $\psi_{h}(t)$ bring the representation

$$
\begin{aligned}
& P_{0}\left(A_{h} f\right)(t)=\left(\int_{-\infty}^{+\infty} f(\tau) G(\tau) d \tau-\frac{\int_{-\infty}^{+\infty} \phi_{h}(\tau) G(\tau) d \tau}{\eta_{h}} \int_{-\infty}^{+\infty} f(t) s_{h}(t) d t+\right. \\
& \left.+\frac{\phi_{h}(0)}{\left(\gamma^{2}-\alpha^{2}\right) \eta_{h}} \int_{-\infty}^{+\infty} f(t) s_{h}(t) d t\right) \iota(t) .
\end{aligned}
$$


Our goal is to find the strong limit $s$ - $\lim _{h \rightarrow+0} A_{h}$. Due to $(26) \lim _{h \rightarrow+0}\left(I-P_{0}\right)\left(A_{h} f\right)(t)$ coincides with $\lim _{h \rightarrow+0}\left(\gamma^{2} I+\widehat{D}_{h}\right)^{-1} f(t)$ in the norm topology of the space $L^{2}(\mathbb{R})$, so $(21)$ brings (note that $\left.P_{0} G(t) \operatorname{Sgn}(t)=0\right)$

$$
\begin{aligned}
& \lim _{h \rightarrow+0}\left(I-P_{0}\right)\left(A_{h} f\right)(t)=\left(I-P_{0}\right) \int_{-\infty}^{+\infty} f(\tau) G(t-\tau) d \tau+ \\
& +\left(\frac{2 \gamma^{2} G(t) \operatorname{Sgn}(t)}{(\gamma-\beta)} \int_{-\infty}^{+\infty} f(t) G(t) \operatorname{Sgn}(t) d t\right) .
\end{aligned}
$$

Simultaneously (20), (23), (25) and (28) bring

$$
\begin{aligned}
& \lim _{h \rightarrow 0} P_{0}\left(A_{h} f\right)(t)=P_{0} \int_{-\infty}^{+\infty} f(\tau) G(t-\tau) d \tau+ \\
& +\left(\frac{-\gamma^{2}}{\left(\gamma^{2}-\alpha^{2}\right)} \int_{-\infty}^{+\infty} f(t) G(t) d t+\frac{1}{\left(\gamma^{2}-\alpha^{2}\right)} f(0)\right) \iota(t) .
\end{aligned}
$$

Let $A_{0}=s-\lim _{h \rightarrow+0} A_{h}$. Then due to (29) and (30)

$$
\begin{aligned}
\left(A_{0} f\right)(t)= & \int_{-\infty}^{+\infty} f(\tau) G(t-\tau) d \tau+\frac{2 \gamma^{2} G(t) \operatorname{Sgn}(t)}{(\gamma-\beta)} \int_{-\infty}^{+\infty} f(t) G(t) \operatorname{Sgn}(t) d t+ \\
& +\left(\frac{-\gamma^{2}}{\left(\gamma^{2}-\alpha^{2}\right)} \int_{-\infty}^{+\infty} f(t) G(t) d t+\frac{1}{\left(\gamma^{2}-\alpha^{2}\right)} f(0)\right) \iota(t)
\end{aligned}
$$

where $f(t) \in L^{2}(\mathbb{R}) \cap \mathbf{C}(\mathbb{R})$ and $\left(A_{0} f\right)(t) \in L_{\sigma_{0}}^{2}(\mathbb{R})$. The space $L^{2}(\mathbb{R}) \cap \mathbf{C}(\mathbb{R})$ has the natural dense embedding in the space $L_{\sigma_{0}}^{2}(\mathbb{R})$. Thanks to this observation one can introduce in $L_{\sigma_{0}}^{2}(\mathbb{R})$ the operator $\hat{A}_{0}$ that initially was defined on $L^{2}(\mathbb{R}) \cap \mathbf{C}(\mathbb{R}) \subset L_{\sigma_{0}}^{2}(\mathbb{R})$ as in $(31)$ and is extended on whole $L_{\sigma_{0}}^{2}(\mathbb{R})$ by continuity. In particular, if $\xi_{n}(t)=e^{-(n t)^{2}}$, then

$$
\left(\hat{A}_{0} \iota\right)(t)=\lim _{n \rightarrow \infty}\left(\hat{A}_{0} \xi_{n}\right)(t)=\frac{1}{\left(\gamma^{2}-\alpha^{2}\right)} \iota(t),
$$

i.e. $\iota(t)$ is an eigenfunction of $\hat{A}_{0}$.

Note that $\hat{A}_{0}$ is not a selfadjoint operator. The latter contradicts the standard frame of Quantum Mechanics (see, for instance, [11]), so we need to construct a larger space where $\hat{A}_{0}$ can be extended to a selfadjoint operator. Let us recall Observation (14). The latter indicates that $\delta(t)$ is a natural candidate to be include in a new larger space. Let $\mathfrak{L}$ be the linear span of $L^{2}(\mathbb{R}) \cap \mathbf{C}(\mathbb{R})$ and $\delta(t)$. One can introduce on $\mathfrak{L}$ a natural partial inner product $[\cdot, \cdot]:[x(t), \delta(t)]=x(0),[x(t), y(t)]=\int_{-\infty}^{\infty} x(t) \overline{y(t)} d t$, where $x(t), y(t) \in L^{2}(\mathbb{R})$. The inner product $[\delta(t), \delta(t)]$ does not defined yet. Let $[\delta(t), \delta(t)]=q$, where $q$ is some real number. Then $\left[\delta(t)-\tau \xi_{n}(t), \delta(t)-\tau \xi_{n}(t)\right]=q-2 \tau+\tau^{2} \frac{\sqrt{2 \pi}}{2 n}$, where $\tau \in \mathbb{R}, \xi_{n}(t)=e^{-(n t)^{2}}$. 
This yields $\left.\left[\delta(t)-\tau \xi_{n}(t), \delta(t)-\tau \xi_{n}(t)\right]\right|_{\tau=\frac{2 n}{\sqrt{2 \pi}}}=q-\frac{2 n}{\sqrt{2 \pi}}$, so the latter is negative if $n$ is big enough. Thus, the quadratic form $[x, x]$ is indefinite on $\mathfrak{L}$ for any $q$, so $\mathfrak{L}$ can be consider as a space with indefinite metric. Notions and results concerning spaces with indefinite metric one can find in [12]. For simplicity we put $q=0$, so

$$
[\delta(t), \delta(t)]=0, \quad[x(t), \delta(t)]=x(0), \quad[x(t), y(t)]=\int_{-\infty}^{\infty} x(t) \overline{y(t)} d t
$$

where $x(t), y(t) \in L^{2}(\mathbb{R})$. Note that the choice $q=0$ is compatible with the approach to Distribution Theory for discontinuous test functions given in [6]. $\mathfrak{L}$ represents a prePontryagin space, where the number of negative squares for the corresponding quadratic form is one. This space can be completed till a Pontryagin space. There are different ways to introduce a Hilbert scalar product, all these ways are topologically equivalent. Let us give one of them. The completion $\mathfrak{P}$ of $\mathfrak{L}$ is presented in the form $\mathfrak{P}=L_{\sigma_{0}}^{2}(\mathbb{R}) \oplus\{\xi \delta(t)\}_{\xi \in \mathbb{C}}$, $\|\delta(t)\|=1$ and the space $L_{\sigma_{0}}^{2}(\mathbb{R})$ conserves its Hilbert structure. Note that $\iota(t) \in L_{\sigma_{0}}^{2}(\mathbb{R}) \subset$ $\mathfrak{P},\|\iota(t)\|=1$ and due to $(33)[\delta, \iota]=1, \quad[\iota, \iota]=0, \quad L_{\sigma_{0}}^{2}(\mathbb{R})=L^{2}(\mathbb{R}) \oplus\{\varsigma \iota(t)\}_{\varsigma \in \mathbb{C}}$. Our goal is to find an extension $C_{0}$ of $\hat{A}_{0}$ in such a way that $\left[C_{0} x, y\right]=\left[x, C_{0} y\right]$ for all $x, y \in \mathfrak{P}$. This property in the case of Pontryagin or Krein spaces (see [12]) is called $J$-selfadjointness. Evidently we need to find $\left(C_{0} \delta\right)(t)$. Thus, due to $(31)$ and $(32)$

$$
\begin{aligned}
& {\left[C_{0} \delta(t), f(t)\right]=\left[\delta(t),\left(\hat{A}_{0} f\right)(t)\right]=\int_{-\infty}^{+\infty} \bar{f}(\tau) G(\tau) d \tau-\frac{\gamma^{2}}{\left(\gamma^{2}-\alpha^{2}\right)} \int_{-\infty}^{+\infty} \bar{f}(t) G(t) d t+} \\
& +\frac{1}{\left(\gamma^{2}-\alpha^{2}\right)}[\delta(t), f(t)],
\end{aligned}
$$

where $f(t) \in L_{\sigma_{0}}^{2}(\mathbb{R})$. Note that $\left[\left(C_{0} \delta\right)(t), \delta(t)\right]$ cannot calculate using $\hat{A}_{0}$, so put $\left[\left(C_{0} \delta\right)(t), \delta(t)\right]=\eta$ with ambiguous $\eta \in \mathbb{R}$. Summarizing these facts we have

$$
C_{0} \delta(t)=\frac{1}{\left(\gamma^{2}-\alpha^{2}\right)} \delta(t)-\frac{\alpha^{2}}{\left(\gamma^{2}-\alpha^{2}\right)} G(t)+\eta \iota(t) \text {. }
$$

Using Equality

$$
\int_{-\infty}^{+\infty} e^{-\alpha|\tau|} G(t-\tau) d \tau=\frac{e^{-\alpha|t|}}{\left(\gamma^{2}-\alpha^{2}\right)}-\frac{2 \alpha G(t)}{\left(\gamma^{2}-\alpha^{2}\right)}
$$

and Representations (31), (32), (34) let us calculate $C_{0}\left(\phi_{0}(t)-\frac{2}{\alpha} \delta(t)\right)$ with $\phi_{0}$ from (14):

$$
C_{0}\left(\phi_{0}(t)-\frac{2}{\alpha} \delta(t)\right)=\frac{1}{\left(\gamma^{2}-\alpha^{2}\right)}\left(\phi_{0}(t)-\frac{2}{\alpha} \delta(t)\right)+\iota(t)\left(\frac{\alpha}{\left(\gamma^{2}-\alpha^{2}\right)} \frac{1}{(\gamma+\alpha)}-\frac{2 \eta}{\alpha}\right) .
$$

If $\left(\frac{\alpha}{\left(\gamma^{2}-\alpha^{2}\right)} \frac{1}{(\gamma+\alpha)}-\frac{2 \eta}{\alpha}\right) \neq 0$ then $\left(\phi_{0}(t)-\frac{2}{\alpha} \delta(t)\right)$ is not an eigenfunction of $C_{0}$ but it is a function adjoin to the eigenfunction $\iota(t)$. This situation does not correspond to postulates 
of Quantum Mechanics [11], therefore $\left(\frac{\alpha}{\left(\gamma^{2}-\alpha^{2}\right)} \frac{1}{(\gamma+\alpha)}-\frac{2 \eta}{\alpha}\right)=0$, so

$$
\begin{aligned}
\left(C_{0} f\right)(t)= & \int_{-\infty}^{+\infty} f(\tau) G(t-\tau) d \tau+\frac{2 \gamma^{2} G(t) \operatorname{Sgn}(t)}{(\gamma-\beta)} \int_{-\infty}^{+\infty} f(t) G(t) \operatorname{Sgn}(t) d t+ \\
& +\left(\frac{-\gamma^{2}}{\left(\gamma^{2}-\alpha^{2}\right)} \int_{-\infty}^{+\infty} f(t) G(t) d t+\frac{1}{\left(\gamma^{2}-\alpha^{2}\right)} f(0)\right) \iota(t), f(t) \in L_{\sigma_{0}}^{2}(\mathbb{R}), \\
C_{0} \delta(t)= & \frac{1}{\left(\gamma^{2}-\alpha^{2}\right)} \delta(t)-\frac{\alpha^{2}}{\left(\gamma^{2}-\alpha^{2}\right)} G(t)+\frac{\alpha^{2}}{2\left(\gamma^{2}-\alpha^{2}\right)(\gamma+\alpha)} \iota(t) .
\end{aligned}
$$

Remark 3. Formulae (36) completely define $J$-selfadjoint operator $C_{0}$. By construction this operator has two eigenfunctions $\iota(t)$ and $\left(\phi_{0}(t)-\frac{2}{\alpha} \delta(t)\right)$ that correspond to the eigenvalue $\frac{1}{\left(\gamma^{2}-\alpha^{2}\right)}$. Simultaneously Remark 2 shows that the function $\psi_{0}(t)=e^{-\beta|t|} \operatorname{Sgn}(t)$, $\psi_{0}(t)=0$ is an eigenfunction for $C_{0}$ corresponding to the eigenvalue $\frac{1}{\left(\gamma^{2}-\beta^{2}\right)}$.

\subsection{A Pontryagin Space Effect}

We use the terms "positive vector", "neutral vector", "non-negative subspace", "maximal non-negative subspace", etc., in the usual way: they are defined with respect to the sign of the quadratic form $[\cdot, \cdot]$ (see [12]). Analogously by the symbol $[\perp]$ we denote the orthogonality of vectors or sets with respect to the inner product $[\cdot, \cdot] . J$-selfadjoint operators in Pontryagin spaces have a key property: any $J$-selfadjoint operator has at least one maximal non-positive invariant subspace. The dimension of maximal non-positive subspace coincides with the range of indefiniteness of the corresponding space.If $\mathcal{L}_{-}$is a maximal non-positive invariant subspace for a $J$-selfadjoint operator $B$ then $J$-orthogonal subspace $\mathcal{L}_{-}^{[\perp]}$ is non-negative, maximal and invariant for $B$. Note that if $\mathcal{L}_{-}$is a negative subspace then $\mathcal{L}_{-}^{[\perp]}$ is positive one. In the case of Pontryagin space the inner product $[\cdot, \cdot]$ restricted on a positive subspace is topologically equivalent to the original Hilbert scalar product. This brings an option to return completely to the postulates of Quantum Mechanics where operators must act in Hilbert spaces.

Due to $(33)\left[\phi_{0}(t)-\frac{2}{\alpha} \delta(t), \phi_{0}(t)-\frac{2}{\alpha} \delta(t)\right]=\int_{-\infty}^{\infty} e^{-2 \alpha|t|} d t-\frac{4}{\alpha}=-\frac{3}{\alpha}$.

Thus, $\phi_{0}(t)-\frac{2}{\alpha} \delta(t)$ is a negative element of $\mathfrak{P}$. Recall (36). One can consider the subspace spanned by $\phi_{0}(t)-\frac{2}{\alpha} \delta(t)$ as a maximal negative subspace $\mathcal{L}_{-}$invariant with respect to $C_{0}$. Let $\mathfrak{N}_{\alpha}$ be the linear span of $\phi_{0}(t)-\frac{2}{\alpha} \delta(t)$ and $\iota(t)$. Put

$$
\vartheta(t)=\iota(t)+\frac{\alpha}{3}\left[\iota(t), \phi_{0}(t)-\frac{2}{\alpha} \delta(t)\right]\left(\phi_{0}(t)-\frac{2}{\alpha} \delta(t)\right)=\iota(t)-\frac{2}{3}\left(\phi_{0}(t)-\frac{2}{\alpha} \delta(t)\right) .
$$

Then $\vartheta(t) \in \mathfrak{N}_{\alpha}, \phi_{0}(t)-\frac{2}{\alpha} \delta(t)[\perp] \vartheta(t), \vartheta(t)$ is an eigenfunction for $C_{0}$ and $[\vartheta(t), \vartheta(t)]=$ $\frac{8}{3 \alpha}+\frac{4}{3 \alpha}=\frac{4}{\alpha}$, i.e. $\mathfrak{N}_{\alpha}$ is an indefinite subspace. Since it is invariant for $C_{0}$, its orthogonal complement $\mathfrak{N}_{\alpha}^{[\perp]}$ is also invariant for $C_{0}$, moreover it is positive. Put $\mathfrak{N}=\mathfrak{N}_{\alpha}^{[\perp]}$. Then $\mathfrak{N}$ can be described as follows 


$$
\mathfrak{N}=\left\{f(t): f(t) \in L_{\sigma_{0}}^{2}(\mathbb{R}), f(0)=\frac{\alpha}{2} \int_{-\infty}^{+\infty} f(t) \phi_{0}(t) d t\right\}
$$

Note that for $f(t), g(t) \in \mathfrak{N}$ the representation $[f(t), g(t)]=\int_{-\infty}^{+\infty} f(t) \overline{g(t)} d t$ takes place, so $\mathfrak{N}$ can be considered as a Hilbert space isometric to $L^{2}(\mathbb{R})$ in the evident sense. Note also (see Remark 2) that $\psi_{0}(t) \in \mathfrak{N}$. If $f(t) \in L_{\sigma_{0}}^{2}(\mathbb{R})$, then it must be defined at zero. For $u(t) \in \mathfrak{P}, u(t)=f(t)+\xi \delta(t), f(t) \in L_{\sigma_{0}}^{2}(\mathbb{R})$ we denote

$$
\vec{u}(t)= \begin{cases}f(t), & \text { if } t \neq 0 \text { and } f(t) \text { is defined at } t \\ 0, & \text { if } t=0,\end{cases}
$$

so (see $(24)) f(t)=\stackrel{\diamond}{f}(t)+f(0) \iota(t)$. For an odd function $f(t)$ evidently $\stackrel{\diamond}{f}(t)=f(t)$, i.e.

$$
f(0)=0 \text { for any odd function } f(t) \in L_{\sigma_{0}}^{2}(\mathbb{R}) \text {. }
$$

Moreover, we assume that all functions from $L^{2}(\mathbb{R})$ are simultaneously functions from $L_{\sigma_{0}}^{2}(\mathbb{R})$ nullified at zero. With slight abuse of Notation $(39)$ we shall use notations like $\overbrace{u^{\prime}}^{\ominus}(t)$ if $\stackrel{\leftrightarrow}{u}(t)$ is absolutely continuous function on $\mathbb{R} \backslash\{0\}$; here and below $u^{\prime}(t)$ and $(\stackrel{\leftrightarrow}{u})^{\prime}(t)$ are treated as a derivatives of generalized functions. Thus, the symbol $\overbrace{u^{\prime}}^{\diamond}(t)$ is defined as follows: $\overbrace{u^{\prime}}^{\ominus}(t)=\overbrace{\dot{u}^{\prime}}^{\ominus}(t)$. By the same way the symbol $\overbrace{u^{\prime \prime}}^{\ominus}(t)$ is introduced.

Our aim now is a calculation of $\left.C_{0}^{-1}\right|_{\mathfrak{N}}$. First, let us find the range $\operatorname{Rg}\left(\left.C_{0}\right|_{\mathfrak{N}}\right)$ of the operator $\left.C_{0}\right|_{\mathfrak{N}}$. Taking into account (36) for $f(t) \in \mathfrak{N}$, (40) and Denotation (39) we have

$$
\begin{aligned}
& C_{0} f(t)=C_{0}\left(\stackrel{\ominus}{f}(t)+\frac{\alpha}{2} \int_{-\infty}^{+\infty} f(t) \phi_{0}(t) d t \cdot \iota(t)\right)=\overbrace{\int_{-\infty}^{+\infty} f(\tau) G(t-\tau) d \tau}^{\ominus}+ \\
& +\overbrace{\frac{2 \gamma^{2}}{\gamma-\beta} G(t) \operatorname{Sgn}(t)}^{\ominus} \int_{-\infty}^{+\infty} f(t) G(t) \operatorname{Sgn}(t) d t)+ \\
& +\iota(t)\left(-\frac{\alpha^{2}}{\left(\gamma^{2}-\alpha^{2}\right)} \int_{-\infty}^{+\infty} f(t) G(t) d t+\frac{\alpha}{2\left(\gamma^{2}-\alpha^{2}\right)} \int_{-\infty}^{+\infty} f(t) \phi_{0}(t) d t\right) .
\end{aligned}
$$

Next, using Equality (35)) one can transform Equality (41) to

$$
C_{0}\left(\grave{f}(t)+\frac{\alpha}{2} \int_{-\infty}^{+\infty} f(t) \phi_{0}(t) d t \cdot \iota(t)\right)=\stackrel{u}{u}(t)+\frac{\alpha}{2} \int_{-\infty}^{+\infty} u(t) \phi_{0}(t) d t \cdot \iota(t)
$$

with

$$
u(t)=\int_{-\infty}^{+\infty} f(\tau) G(t-\tau) d \tau+\frac{2 \gamma^{2}}{\gamma-\beta} G(t) \operatorname{Sgn}(t) \int_{-\infty}^{+\infty} f(t) G(t) \operatorname{Sgn}(t) d t
$$


Lemma 1. $u(t) \in \operatorname{Rg}\left(\left.C_{0}\right|_{\mathfrak{N}}\right)$ if and only if simultaneously

1) the restrictions $\left.u(t)\right|_{(-\infty, 0)},\left.u^{\prime}(t)\right|_{(-\infty, 0)},\left.u(t)\right|_{(0,+\infty)}$, and $\left.u^{\prime}(t)\right|_{(0,+\infty)}$ are absolutely continuous;

2) $u(t), \overbrace{u^{\prime}}^{\diamond}(t), \overbrace{u^{\prime \prime}}^{\diamond}(t) \in L_{\sigma_{0}}^{2}(\mathbb{R})$;

3) $\beta(u(-0)-u(+0))=u^{\prime}(-0)+u^{\prime}(+0)$;

4) $u^{\prime}(-0)=u^{\prime}(+0)$;

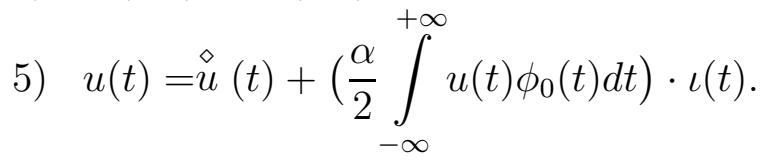

If $u(t) \in \operatorname{Rg}\left(\left.C_{0}\right|_{\mathfrak{N}}\right)$, then $C_{0}^{-1}\left(\grave{u}(t)+\frac{\alpha}{2} \int_{-\infty}^{+\infty} u(t) \phi_{0}(t) d t \cdot \iota(t)\right)=$

$=\overbrace{-u^{\prime \prime}(t)+\gamma^{2} u(t)}^{\ominus}+\frac{\alpha}{2} \int_{-\infty}^{+\infty} \overbrace{\left(-u^{\prime \prime}(t)+\gamma^{2} u(t)\right)}^{\ominus}(t) \phi_{0}(t) d t \cdot \iota(t)$,

Proof. The statement of Lemma 1 follows mainly from Remark 2, its proof and Formulae (41), (42), (43). Note that, generally speaking, $u(t)$ contains a jump at zero, so $u^{\prime}(t)$ represents a generalized function with a singularity at zero, therefore instead of $u^{\prime}$ the function $\overbrace{u^{\prime}}^{\ominus}(t)$ was used, etc.

Now we fix a maximal positive subspace $\mathcal{L}_{+}$invariant with respect to $C_{0}$. Such a subspace is not uniquely defined. We put $\mathcal{L}_{+}=\mathfrak{N} \dot{+}\{\zeta \varrho(t)\}_{\zeta \in \mathbb{C}}$, where (see $\left.(37)\right) \varrho(t)=$ $-\frac{3}{2} \vartheta(t)=\phi_{0}(t)-\frac{2}{\alpha} \delta(t)-\frac{3}{2} \iota(t)$.

Remark 4. The subspaces $\mathcal{L}_{+}$is a $J$-orthogonal complement of $\phi_{0}(t)-\frac{2}{\alpha} \delta(t)$ and can be presented as $\left\{u(t)=\stackrel{u}{u}(t)+\frac{\alpha}{2}\left(\int_{-\infty}^{+\infty} \grave{u}(t) \phi_{0}(t) d t-\zeta\right) \cdot \iota(t)-\zeta \delta(t)\right\}_{\zeta \in \mathbb{C}, \grave{u}(t) \in L^{2}(\mathbb{R})}$.

Theorem 1. $u(t) \in \operatorname{Rg}\left(\left.C_{0}\right|_{\mathcal{L}_{+}}\right)$if and only if simultaneously

1) the restrictions $\left.u(t)\right|_{(-\infty, 0)},\left.u^{\prime}(t)\right|_{(-\infty, 0)},\left.u(t)\right|_{(0,+\infty)}$, and $\left.u^{\prime}(t)\right|_{(0,+\infty)}$ are absolutely continuous;

2) $\stackrel{\diamond}{u}(t), \overbrace{u^{\prime}}^{\ominus}(t), \overbrace{u^{\prime \prime}}^{\ominus}(t) \in L^{2}(\mathbb{R})$;

3) $\beta(u(-0)-u(+0))=u^{\prime}(-0)+u^{\prime}(+0)$;

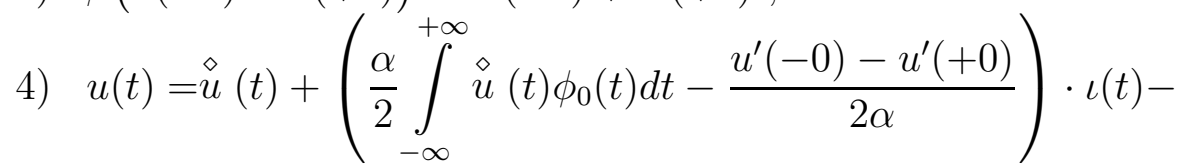

$$
-\frac{u^{\prime}(-0)-u^{\prime}(+0)}{\alpha^{2}} \delta(t)
$$


If $u(t) \in \operatorname{Rg}\left(\left.C_{0}\right|_{\mathcal{L}_{+}}\right)$, then

$$
\begin{aligned}
& C_{0}^{-1} u(t)=\overbrace{-u^{\prime \prime}+\gamma^{2} u}^{\ominus}(t)-\frac{u^{\prime}(-0)-u^{\prime}(+0)}{\alpha^{2}}\left(\gamma^{2}-\alpha^{2}\right) \delta(t)+ \\
& +(\frac{\alpha}{2} \int_{-\infty}^{+\infty} \overbrace{\left(-u^{\prime \prime}+\gamma^{2} u\right)}^{\ominus}(\tau) \phi_{0}(\tau) d \tau-\frac{u^{\prime}(-0)-u^{\prime}(+0)}{2 \alpha}\left(\gamma^{2}-\alpha^{2}\right)) \iota(t) .
\end{aligned}
$$

Proof. First, $\operatorname{Rg}\left(\left.C_{0}\right|_{\mathcal{L}_{+}}\right)=\operatorname{Rg}\left(\left.C_{0}\right|_{\mathfrak{N}}\right)[\dot{+}]\{\zeta \varrho(t)\}_{\zeta \in \mathbb{C}}$. Note that $\varrho(t)$ satisfies $(45)$ and $(44)$ is a particular case of $(45)$, so for any $u(t) \in \operatorname{Rg}\left(\left.C_{0}\right|_{\mathcal{L}_{+}}\right)$Conditions (45) are fulfilled. Now let us consider the inverse statement. Take $u(t)$ satisfying $(45)$ and put $v(t)=u(t)-$ $\frac{u^{\prime}(-0)-u^{\prime}(+0)}{2 \alpha} \varrho(t)$. Then $v^{\prime}(-0)=\frac{u^{\prime}(-0)+u^{\prime}(+0)}{2}=v^{\prime}(+0)$. Due to Lemma $1 v(t) \in$ $\operatorname{Rg}\left(\left.C_{0}\right|_{\mathfrak{N}}\right)$, so $C_{0}^{-1} u(t)=C_{0}^{-1} v(t)+\frac{u^{\prime}(t-0)-u^{\prime}(t+0)}{2 \alpha} C_{0}^{-1} \rho(t)=\overbrace{-v^{\prime \prime}+\gamma^{2} v}^{\ominus}(t)+$

$$
+\frac{\alpha}{2} \int_{-\infty}^{+\infty} \overbrace{\left(-v^{\prime \prime}+\gamma^{2} v\right)}^{\ominus}(\tau) \phi_{0}(\tau) d \tau \cdot \iota(t)+\frac{u^{\prime}(t-0)-u^{\prime}(t+0)}{2 \alpha}\left(\gamma^{2}-\alpha^{2}\right) \varrho(t) .
$$

Next, $\overbrace{\left(-v^{\prime \prime}+\gamma^{2} v\right)}^{\ominus}(t)=\overbrace{\left(-u^{\prime \prime}+\gamma^{2} u\right)}^{\ominus}(t)-\frac{\left(u^{\prime}(-0)-u^{\prime}(+0)\right)\left(\gamma^{2}-\alpha^{2}\right)}{2 \alpha} \overbrace{\phi_{0}}^{\diamond}(t)=$. $\overbrace{\left(-u^{\prime \prime}+\gamma^{2} u\right)}^{\ominus}(t)-\frac{\left(u^{\prime}(-0)-u^{\prime}(+0)\right)\left(\gamma^{2}-\alpha^{2}\right)}{2 \alpha}\left(\phi_{0}(t)-\iota(t)\right)$. The latter yields $(46)$.

Corollary 1. If $u(t) \in \operatorname{Rg}\left(\left.C_{0}\right|_{\mathcal{L}_{+}}\right)$, then

$$
\begin{aligned}
& \left(C_{0}^{-1}-\gamma^{2} I\right) u(t)=-\overbrace{u^{\prime \prime}}^{\diamond}(t)+\left(u^{\prime}(-0)-u^{\prime}(+0)\right) \delta(t)- \\
& -\frac{\alpha}{2}(\int_{-\infty}^{+\infty} \overbrace{u^{\prime \prime}}^{\ominus}(\tau) \phi_{0}(\tau) d \tau-\left(u^{\prime}(-0)-u^{\prime}(+0)\right)) \iota(t),
\end{aligned}
$$

so the operator $\left(C_{0}^{-1}-\gamma^{2} I\right)$ can be consider as a selfadjoint extension on a larger space of the operator $D_{0}$ from (1).

Taking into account the statement of Theorem 1 it seems natural to consider (generalized) functions of the type

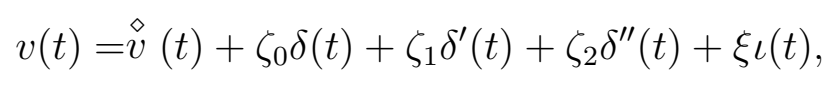

where $\zeta_{0}, \zeta_{1}, \zeta_{2}, \xi \in \mathbb{C}, \stackrel{\diamond}{v}(t) \in L^{2}(\mathbb{R})$. For the function $v(t)$ from $(48)$ we put $\stackrel{v}{v}(t)=\stackrel{\diamond}{v}$ $(t)+\zeta_{0} \delta(t)+\zeta_{1} \delta^{\prime}(t)+\zeta_{2} \delta^{\prime \prime}(t)$. Using the latter notation one can re-write $u(t)$ from (45.4) as (compare with (44.5)!) $u(t)=\sqrt{ }(t)+\frac{\alpha}{2}\left(\int_{-\infty}^{+\infty} \vec{v}(\tau) \phi_{0}(\tau) d \tau\right) \iota(t)$. Let us return to 
Formula (15). Strictly speaking in (15) we cannot pass to limit by $h \rightarrow 0$, but from some heuristic point of view we can say that $\widehat{D}_{h} \rightarrow \widehat{D}_{0}$, where

$$
\widehat{D}_{0} y(t)=-y^{\prime \prime}(t)-\frac{\delta^{\prime \prime}(t)\left(y^{\prime}(-0)-y^{\prime}(+0)\right)}{\alpha^{2}}-\frac{\delta^{\prime}(t)\left(y^{\prime}(-0)+y^{\prime}(+0)\right)}{\beta} .
$$

For instance, one can consider the limit in question as the weak limit of generalized functions, where $y(t)$ is an arbitrary function of bounded variation. As it is clear, the operator $\widehat{D}_{0}$ is not well defined as a selfadjoint operator because its domain and a corresponding Hilbert space are not evident. Let us consider $u(t) \in \operatorname{Rg}\left(\left.C_{0}\right|_{\mathcal{L}_{+}}\right)$. If $u(t)$ is treated as a generalized function, then $\iota(t)$ is equivalent to zero and

$$
u^{\prime \prime}(t)=\overbrace{u^{\prime \prime}}^{\ominus}(t)-\left(u^{\prime}(-0)-u^{\prime}(+0)\right) \delta(t)-\frac{u^{\prime}(-0)+u^{\prime}(+0)}{\beta} \delta^{\prime}(t)-\frac{u^{\prime}(-0)-u^{\prime}(+0)}{\alpha^{2}} \delta^{\prime \prime}(t) .
$$

This means that the function $-u^{\prime \prime}(t)-\frac{\delta^{\prime \prime}(t)\left(u^{\prime}(-0)-u^{\prime}(+0)\right)}{\alpha^{2}}-\frac{\delta^{\prime}(t)\left(u^{\prime}(-0)+u^{\prime}(+0)\right)}{\beta}$ coincides with the function $\overbrace{\left(C_{0}^{-1}-\gamma^{2} I\right) u}^{v}(t)$ defined by (47). Thus, we can re-define the operator $\widehat{D}_{0}$ from $(49)$ as a selfadjoint operator $\breve{D}_{0}$ in the Hilbert space $\mathcal{L}_{+}$with the scalar product $[\cdot, \cdot \cdot]$ putting $\breve{D}_{0}=\left(C_{0}^{-1}-\gamma^{2} I\right)$. A detailed description yields $\left(y(t) \in \operatorname{Rg}\left(\left.C_{0}\right|_{\mathcal{L}_{+}}\right)\right)$

$$
\begin{aligned}
& \breve{D}_{0} y(t)=-\overbrace{y^{\prime \prime}}^{\sqrt{ }}(t)-\frac{\delta^{\prime \prime}(t)\left(y^{\prime}(-0)-y^{\prime}(+0)\right)}{\alpha^{2}}-\frac{\delta^{\prime}(t)\left(y^{\prime}(-0)+y^{\prime}(+0)\right)}{\beta}- \\
& -\frac{\alpha}{2}\left(\int_{-\infty}^{+\infty}(\overbrace{y^{\prime \prime}}^{\sqrt{ }}(t)+\frac{\delta^{\prime \prime}(t)\left(y^{\prime}(-0)-y^{\prime}(+0)\right)}{\alpha^{2}}+\frac{\delta^{\prime}(t)\left(y^{\prime}(-0)+y^{\prime}(+0)\right)}{\beta}) \phi_{0}(t) d t\right) \iota(t) .
\end{aligned}
$$

The operator $\breve{D}_{0}$ represents a new type of the Hamiltonian with one-point interaction that involves $\delta^{\prime \prime}$ singularity. It has two negative eigenvalues $-\alpha^{2}$ and $-\beta^{2}$ with, respectively, the eigenfunctions $\rho(t)$ and $\psi_{0}(t)$. If $\alpha=\beta$, the operator $\breve{D}_{0}$ has simultaneously symmetric and anti-symmetric bound states, the eigenfunction $\rho(t)+\psi_{0}(t)$ is nullified at $t<0$ and the eigenfunction $\rho(t)-\psi_{0}(t)$ is nullified at $t>0$, so the Hamiltonian in question shows an effect of spontaneous symmetry breaking.

\section{Conclusions}

We analyzed here a quantum interaction based in two shifted Dirac delta-functions with appropriated coefficients ensuring the corresponding Hamiltonian to be selfadjoint. To get this we used a method of selfadjoint extensions for symmetric operators. Among the permissible boundary conditions there exists a class of them that leaves the Hamiltonian invariant under parity transformations. Among this class we stayed with those which determine a non-local interaction and called this phenomenon an entanglement of boundary conditions. In that situation the ground state is degenerate and there exist 
eigenstates with the wave function situated on one side of the interaction zone, being zero on its complement, define the left and right handed states respectively. This effect is basically generated by finite coupling constants in the derivative in the two shifted Dirac distributions, in distinction to the case of a local interaction where the only possibility to have spontaneous symmetry breaking occurs for an infinitely high and thick barrier.

The main contribution of the paper consists in the study from different points of view on a limit pass from two-point interaction to one-point one. In particular, a selfadjoint extension of a limit differential operator to a larger Pontryagin space permits to preserve an option of degenerate ground state, moreover, the corresponding Hamiltonian contains the second derivative of delta-function that is a new effect.

\section{References}

1. Restuccia A., Sotomayor A., Strauss V. Non-Local Interactions in Quantum Mechanics Modelled by Shifted Dirac Delta Functions. Journal of Physics: Conference Series, 2016, no. 1043,9 p.

2. Restuccia A., Sotomayor A., Strauss V. On a Model of Spontaneous Symmetry Breaking in Quantum Mechanics. Bulletin of South Ural State University. Mathematical Modelling, Programming and Computer Software, 2020, vol. 13, no. 3, pp. 5-16. DOI: $10.14529 / \mathrm{mmp} 200301$

3. Burrau O. Berechnung des Energiewertes des Wasserstoffmolekel-Ions $\left(H_{+}^{2}\right)$ im Normalzustand. Naturwissenschaften, 1927, vol. 15, no. 1, pp. 16-17. DOI: $10.1007 / \mathrm{BF} 01504875$

4. Kronig R. de L., Penney W.G. Quantum Mechanics of Electrons in Crystal Lattices. Proceedings of the Royal Society, 1931, vol. 130A, pp. 499-513. DOI 10.1098/rspa.1931.0019

5. Albeverio S., Gesztesy F., Hoegh-Kron R., Holden H. Solvable Models in Quantum Mechanics. White River Junction: Chelsea Publishing, 2004.

6. Kurasov P. Distribution Theory for Discontinuous Test Functions and Differential Operators with Generalized Coefficients. Journal of Mathematical Analysis and Applications, 1996, no. 201, pp. 297-323.

7. Zurek W.H. Decoherence, Einselection, and the Quantum Origins of the Classical. Reviews of Modern Physics, 2003, vol. 75, no. 3, pp. 715-776. DOI: 10.1103/RevModPhys.75.715

8. Weinberg S. Lectures on Quantum Mechanics. Cambridge, Cambridge University Press, 2012.

9. Mal'cev A.I. Foundations of Linear Algebra. Freeman and Company, San Francisco, 1963.

10. Kurasov P., Luger A. Reflectionless Potentials and Point Interactions in Pontryagin Spaces. Letters in Mathematical Physics, 2005, vol. 73, pp. 109-122. DOI: 10.1007/s11005-005-0002-1

11. Yariv, A. An Introduction Theory to Theory and Applications of Quantum Mechanics, New York, Wiley and Sons, 1982.

12. Azizov T.Ya., Iokhvidov I.S. Linear Operators in Spaces with Indefinite Metric, New York, Wiley and Sons, 1989.

Received June 26, 2020 


\title{
О ПРЕДЕЛЬНОМ ПЕРЕХОДЕ ОТ ДВУХТОЧЕЧНОГО К ОДНОТОЧЕЧНОМУ ВЗАИМОДЕЙСТВИЮ В ОДНОМЕРНОЙ КВАНТОВО-МЕХАНИЧЕСКОЙ ПРОБЛЕМЕ, ПОРОЖДАЮЩЕЙ СПОНТАННОЕ РАЗРУШЕНИЕ СИММЕТРИИ
}

\author{
A. Рестусия ${ }^{1,2}$, A. Сотомайор ${ }^{1}$, B.A. Штраус С,3 $^{2,3}$ \\ ${ }^{1}$ Университет Антофагасты, г. Антофагаста, Чили \\ ${ }^{2}$ Университет Симон Боливар, г. Каракас, Венесуэла \\ ${ }^{3}$ Ульяновский государственный педагогический университет, г. Ульяновск, \\ Российская Федерация
}

Исследуется спонтанное нарушение симметрии в одномерной квантовомеханичесой проблеме с сингуляным потенциалам, содержащим сдвинутые дельта-функции и их производные. С математической точки зрения при этом используется метод самосопряжённых расширений симметрического дифференциального оператора, заданного на гладких функциях с интегрируемым квадратом модуля, обнуляющихся вместе со своей первой производной в двух внутренних точках вещественной прямой. Как хорошо известно, последний подход приводит к двухточечной краевой задаче с внутренней границей. Мы находим резольвенту для таких расширений и оцениваем её поведение при изменении положения указанных точек. Область определения подобных расширений может содержать функции, терпящие разрыв и/или имеющие разрывную производную в точках, указанных выше, последнее обычно интерпретируется как присутствие взаимозависимых (сцепленных) сингулярным потенциалов (таких, как сдвиг $\delta$-функции Дирака и её первая производная), сосредоточенных в тех же точках Наша цель - найти связанные состояния с нарушенной симметрией. Для частного случая взаимозависимых граничных условий мы доказываем существование связанного состояния, приводящего к спонтанному нарушению симметрии. Показано, что в терминах пространства Понтрягина возможно сохранения таких состояний в предельном случае, когда расстояние между указанными выше точками обнуляется. Этот результат затем переформулируется в терминах расширенного гильбертова пространства.

Ключевые слова: самосопряженные расширения симметрического дифберенциального оператора; резольвента; решение волнового уравнения: связанные состояния; спонтанное нарушение симметрии; пространства Понтрягина.

Альваро Рестусия, доктор наук $(\mathrm{PhD})$, профессор, вице-ректор по науке, департамент физики, университет Антофагасты (г. Антофагаста, Чили); професcop, департамент физики, университет Симон Боливар (г. Каракас, Венесуэла), alvaro.restuccia@uantof.cl.

Адриан Сотомайор, доктор наук $(\mathrm{PhD})$, доцент, департамент математики, университет Антофагасты (г. Антофагаста, Чили), adrian.sotomayor@uantof.cl.

Владимир Абрамович Штраус, доктор физико-математических наук, профессор, кафедра высшей математики, Ульяновский государственный педагогический университет (г. Ульяновск, Российская Федерация); профессор, департамент чистой и прикладной математики, университет Симон Боливар (г. Каракас, Венесуэла), vstrauss@mail.ru.

Поступила в редакцию 26 июня 2020 г. 